\title{
Earthly powers
}

\author{
A careful analysis of data obtained from the IceCube telescope in Antarctica shows that atmospheric neutrinos can \\ be used as a tomographic probe of the Earth.
}

$\mathrm{N}$ eutrinos are notoriously hard to observe: only $0.001 \%$ of those passing through Earth interact with it - making the chance of detecting a neutrino interaction in a particle detector on the order of one per trillion. One would therefore not expect these particles to be the best available probe for obtaining geophysical information regarding our planet's internal structure. As an analysis by Andrea Donini and colleagues published in this issue of Nature Physics now shows ${ }^{1}$, however, one would be wrong in jumping to this conclusion.

It turns out that the Earth becomes opaque to neutrinos whose energy exceeds $10 \mathrm{TeV}$ : roughly speaking, the diameter of the Earth represents one absorption length for a neutrino with an energy of $25 \mathrm{TeV}$. Moreover, neutrinos on such energy scales are produced in collisions of cosmic rays with nuclei in the Earth's atmosphere. Since their absorption depends on their energy and the distance they have travelled, it is easy to imagine measuring the Earth's density profile by studying the distribution of $\mathrm{TeV}$ atmospheric neutrinos as they pass through our planet.

Indeed, the idea of neutrino tomography is not new and was proposed as long ago as the $1970 \mathrm{~s}^{2,3}$. The problem, of course, was the detection of the atmospheric neutrinos themselves. Thankfully, the advent of largescale neutrino detectors such as the IceCube telescope located in Antarctica has now overcome this issue: significant datasets of the detections of $\mathrm{TeV}$ atmospheric neutrinos have been recorded ${ }^{4,5}$ and, crucially, made publicly available ${ }^{6}$. As Véronique Van Elewyck describes in her News and Views ${ }^{7}$, all of this allowed Donini and colleagues ${ }^{1}$ - who are not directly associated with the IceCube collaboration - to determine the mass of the Earth and its core, its moment of inertia, and indeed establish that the core is more dense than the mantle.

A critic might question what all the fuss is about: after all, this proof of concept is in line with numerical predictions ${ }^{8}$ and, from a geological perspective, we are hardly learning anything new about the structure of the Earth. But consider this: unlike conventional gravimetric methods currently used in geophysics, the neutrino tomography approach relies solely on the knowledge of weak interactions and of nucleon masses - it is completely distinct, both conceptually and methodologically.

The authors estimate that ten more years of IceCube data will dramatically reduce the statistical errors in their analysis, by which time other neutrino detectors such as KM3NeT (currently under construction in the Mediterranean Sea) will be online, and important geophysical questions could be addressed, such as obtaining an independent verification of the density discontinuities at the core-mantle boundary. Could neutrino tomography turn out to be the geophysical equivalent of magnetic resonance imaging of animal tissue? Physicists and engineers are known to like a challenge, so why not?

Published online: 2 January 2019

https://doi.org/10.1038/s41567-018-0403-6

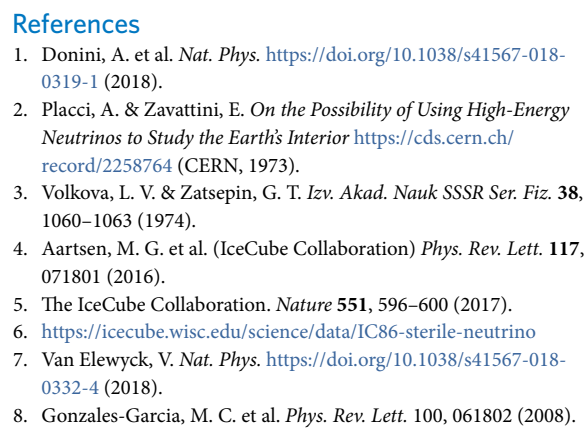

1. Donini, A. et al. Nat. Phys. https://doi.org/10.1038/s41567-0180319-1 (2018) record/2258764 (CERN, 1973).

3. Volkova, L. V. \& Zatsepin, G. T. Izv. Akad. Nauk SSSR Ser. Fiz. 38,

Aartsen, M. G. et al. (IceCube Collaboration) Phys. Rev. Lett. 117

6. https:/icecube wiscedu/sience/data/IC86-sterile-neutrino

7. Van Elewyck, V. Nat. Phys. https://doi.org/10.1038/s41567-018-

8. Gonzales-Garcia, M. C. et al. Phys. Rev. Lett. 100, 061802 (2008).

\section{One to watch}

This month marks the launch of Nature Reviews Physics, the newest addition to the Nature Reviews stables.

W here would we be without the review article? Anyone new to a field will have a favourite tome - dog-eared, if only digitally - that clues them into the basics and conveys all the wisdom of those already in the know. But the review article only appeared in the nineteenth century, two centuries after the first scientific journals surfaced. And the format itself has evolved into a many and varied thing. The launch of Nature Reviews Physics offers the next mutation: an exciting forum for physicists - and the introduction of three new types of review.

Nature Reviews titles first appeared in 2000, offering a short format that championed readability and aesthetic appeal. Nature Physics took up the format, publishing up to ten reviews a year, alongside commentary offering more opinionated perspectives. But dedicated Nature Reviews journals were largely for the benefit of life scientists, until 2016 saw the launch of Nature Reviews Materials, followed in 2017 by Nature Reviews Chemistry, bringing the short-format review to the realm of the physical sciences.

And now, Nature Reviews Physics continues the evolution of the review article by pioneering three new formats. The Technical Review promises to be a one-stop shop for state-of-the-art techniques in a field, a resource offering comprehensive information about a key method to newcomers and experts alike. These details are all too often lost, and Nature Reviews Physics intends to change that - preserving the lessons learned by that brilliant student who hightailed it to Wall Street before the print on her thesis was dry.

Traditional reviews, in a format familiar to our readers, also feature in Nature Reviews Physics, and forthcoming issues will introduce two additional types of review: Roadmaps and Expert Recommendations. Together they will complement our review material and offer physicists a new medium for surveying their most important advances. Take a look online at https://www.nature.com/ natrevphys and follow our youngest sibling on Twitter@NatRevPhys.

Published online: 2 January 2019 https://doi.org/10.1038/s41567-018-0404-5 